\title{
Grating coupler integrated photodiodes for plasmon resonance based sensing
}

\author{
Burak Turker, ${ }^{* a b c}$ Hasan Guner, ${ }^{* a}$ Sencer Ayas, ${ }^{a}$ Okan O. Ekiz, ${ }^{a}$ Handan Acar, ${ }^{a}$ Mustafa O. Guler ${ }^{a}$ \\ and Aykutlu Dâna ${ }^{a}$
}

\author{
Received 8th June 2010, Accepted 15th September 2010 \\ DOI: 10.1039/c0Ic00081g
}

In this work, we demonstrate an integrated sensor combining a grating-coupled plasmon resonance surface with a planar photodiode. Plasmon enhanced transmission is employed as a sensitive refractive index (RI) sensing mechanism. Enhanced transmission of light is monitored via the integrated photodiode by tuning the angle of incidence of a collimated beam near the sharp plasmon resonance condition. Slight changes of the effective refractive index (RI) shift the resonance angle, resulting in a change in the photocurrent. Owing to the planar sensing mechanism, the design permits a high areal density of sensing spots. In the design, absence of holes that facilitate resonant transmission of light, allows an easy-to-implement fabrication procedure and relative insensitivity to fabrication errors. Theoretical and experimental results agree well. An equivalent long-term RI noise of $6.3 \times 10^{-6}$ $R I U / \sqrt{H z}$ is obtained by using an $8 \mathrm{~mW}$ He-Ne laser, compared to a shot-noise limited theoretical sensitivity of $5.61 \times 10^{-9} \mathrm{RIU} / \sqrt{\mathrm{Hz}}$. The device features full benefits of grating-coupled plasmon resonance, such as enhancement of sensitivity for non-zero azimuthal angle of incidence. Further sensitivity enhancement using balanced detection and optimal plasmon coupling conditions are discussed.

\section{Introduction}

Surface plasmon resonance (SPR) can be exploited as a sensitive refractive index (RI) sensing mechanism and SPR is one of the most common label-free biosensor technologies with a vast variety of applications in biology, food safety, medical diagnostics, agricultural and environmental monitoring. ${ }^{1}$ The main concern in the development of SPR is geared toward designing easy to fabricate, cheap and sensitive biosensors with high integration densities.

SPR sensors mainly differ in the optical platforms they are based on. Prism couplers ${ }^{2,3}$ result in comparable higher sensitivity values ${ }^{4}$ than grating assisted coupling in plasmon resonance excitation. However, the bulky nature of prisms makes them unsuitable for applications in integrated sensing devices. Waveguide couplers ${ }^{5}$ employing fibres appear to present the highest degree of miniaturization in SPR sensors, but require expensive high index prisms for the resonant coupling between a surface plasmon and a waveguide mode and suffer from instable sensitivity responses due to deformations in the fibre. Due to their compact size, planarity and stability, grating couplers ${ }^{6}$ have benefits in integrated applications.

In this work, we present a device that eliminates the traditional far field detection (CCD/CMOS detector) mechanism ${ }^{7,8}$ by using an integrated photodetector design. The device structure is illustrated in Fig. 1a and Fig. 1b. The detection mechanism

${ }^{a}$ UNAM, Institute of Materials Science and Nanotechnology, Bilkent University, Ankara, 06800, Turkey. E-mail: bturker@aku.edu.tr; hguner@bilkent.edu.tr

${ }^{b}$ Department of Biomedical Engineering, Afyon Kocatepe University, Afyon, 03200, Turkey

${ }^{c}$ Department of Electronics and Communication Engineering, Ylldiz Technical University, Istanbul, 34349, Turkey works by monitoring the plasmon enhanced transmission of light through a thin metal grating that acts as the plasmon resonance sensing surface. Integrating the photo-diode substrate below the grating structure enables the planar geometry and compact integrity of the sensor. As in other SPR sensors, the resonant shifts are only affected by the changes of optical properties within the close vicinity of the surface.

Transmission of electromagnetic waves through thin metal films via plasmons has been widely studied in the literature. ${ }^{9}$ Plasmon enhanced transmission through sub-wavelength holes, ${ }^{10,11}$ nano-hole arrays ${ }^{12,13}$ or periodically corrugated metal surfaces ${ }^{14}$ have been investigated theoretically and experimentally. ${ }^{15}$ Although plasmon enhancement through nano-hole arrays appears to offer agreeable sensitivity, their realization requires fabrication of holes with high uniformity. Despite the localized nature of plasmon modes in such sub-wavelength aperture arrays and resulting sensitivity to refractive index changes, the overall sensitivity of plasmonic sensors also depends on how sharp the resonances are. Due to fundamental relations between the Fourier components of spatial structure of the surfaces and the SPR, it is difficult to achieve sharp and deep resonances using hole arrays in a simple low-cost fabrication process. In contrast, our approach uses a grating to obtain a plasmonic enhancement of transmission through the silvermetallized grating structures. Lack of holes in our design simplifies the fabrication procedure and increases repeatability. Utilizing a bi-harmonic grating topography ${ }^{16,17,18}$ rather than a sinusoidal one, observation of higher order resonant coupling is also possible in our devices. The bi-harmonic grating structure allows better excitation of the second order resonance ${ }^{18,19,20}$ which also produces an enhancement of light transmission. Such multiple resonances allow SPR detection at a fixed angle of incidence (AOI) for multiple different wavelengths. 
(a)

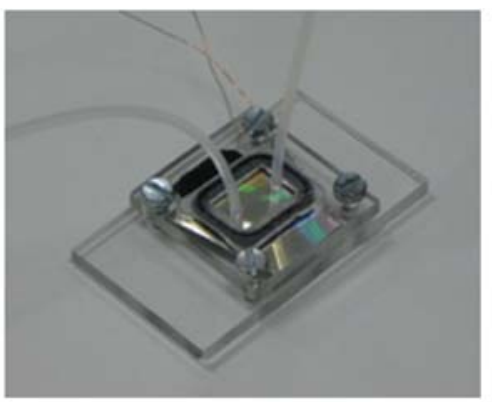

(b)
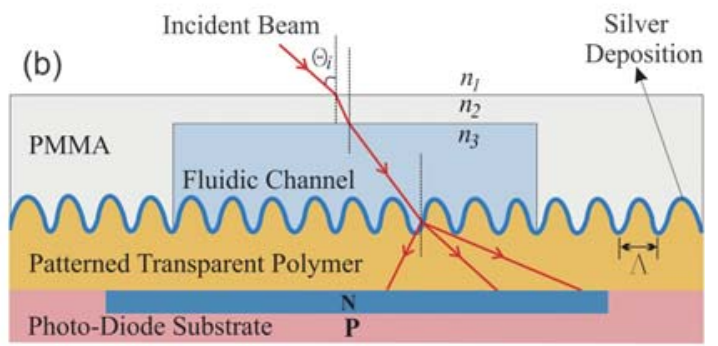

(c)

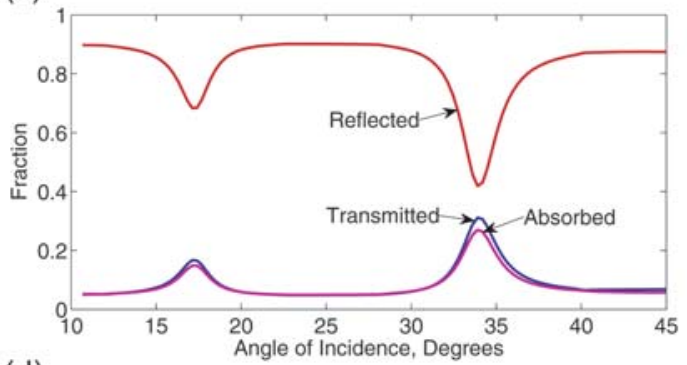

(d)

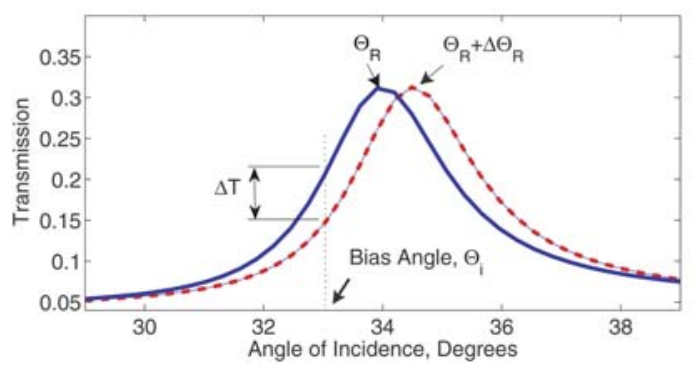

Fig. 1 (a): Photograph of the sensor device with a PMMA fluidic chamber and in/outlet tubing. (b): Cross-sectional schematic view of the device structure. (c): Simulation results of the reflection, transmission and absorption data for TM polarization in the case of a $30 \mathrm{~nm}$ deep sinusoidal grating, deposited with a $45 \mathrm{~nm}$ thick Ag layer and illuminated by a 632.8 $\mathrm{nm}$ light source. The working medium is water with $n_{\mathrm{d}}=1.33$. (d): The operational principle of the device is schematically shown. The illumination is kept at a fixed angle $\Theta_{i}$ and a small perturbation $\left(\Delta \Theta_{R}\right)$ of the resonance condition $\left(\Theta_{\mathrm{R}}\right)$ results in a change $(\Delta \mathrm{T})$ in the transmitted intensity.

\section{Theoretical aspects of grating coupling to the plasmon mode and estimation of SPR sensitivity}

The momentum mismatch between the in-plane wave vector of impinging photons and the wave vector component of the excited surface plasmons disables a natural momentum coupling at planar surfaces. On a smooth planar interface of a metal and a dielectric, the surface plasmon polariton (SPP) wave vector,
$k_{S P P}$, is found to be always greater than the wave vector of incident light in air, $k_{0}$, as given by

$$
k_{S P P}=\frac{\omega}{c} \sqrt{\frac{\varepsilon_{m} \varepsilon_{d}}{\varepsilon_{m}+\varepsilon_{d}}} \geq k_{0}=\frac{\omega}{c} \sqrt{\varepsilon_{d}}
$$

where $\varepsilon_{m}$ is the permittivity of the metal layer and $\varepsilon_{d}$ is that of the dielectric layer (fluid). One of the techniques for satisfying this energy and momentum match condition is to employ grating schemes. The presence of a metal planar surface that is patterned with a shallow grating of grooves with some periodicity modifies eqn (1) as follows

$$
k_{S P P}=m \frac{2 \pi}{\Lambda}+k_{0, \text { InPlane }}
$$

where $m$ is an integer denoting the diffraction order, $k_{0, \text { InPlane }}=$ $k_{0} \operatorname{Sin}\left(\Theta_{R}\right)$ is the in-plane vector of the incident light and $\Lambda$ is the grating period. And thus a plane wave approaching the surface can resonantly couple to the plasmonic excitation at a particular angle of incidence defined by

$$
\Theta_{R}=\arcsin \left[\left(\operatorname{Re} \sqrt{\frac{\varepsilon_{m}}{\varepsilon_{m}+\varepsilon_{d}}}\right)-\frac{m \lambda}{\sqrt{\varepsilon_{d}} \Lambda}\right]
$$

If the measurement is performed inside a fluidic channel with a transparent planar top window, the incidence angle $\Theta_{\mathrm{i}}$ inside the fluidic chamber will be different than that in air, and must be corrected according to Snell's law.

When an organic thin film is applied to the surface the change in the plasmon wave vector ${ }^{21}$ can be approximated by

$$
\delta k_{S P P} \cong \frac{k_{S P P}^{3}}{k_{0}^{2} n_{d}^{3}}\left[1-\exp \left(-2 \gamma_{d} h\right)\right] \Delta n
$$

where $\gamma_{d}=i k_{0} \varepsilon_{d} / \sqrt{\left(\varepsilon_{m}+\varepsilon_{d}\right)}$, and the plasmon mode field penetration depth into the dielectric is $L_{\mathrm{PD}}=1 / \operatorname{Re}\left\{\gamma_{\mathrm{d}}\right\}$. Also, $\Delta \mathrm{n}$ is the refractive index difference of the fluid and the organic thin film and $\mathrm{h}$ is the thickness of the organic thin film. In the presence of an organic film thickness $h \ll L_{\mathrm{PD}}$, the resonance angle shift can be approximated through eqn (2), (3) and (4) as

$$
\delta \Theta_{R}=\frac{k_{S P P}^{3} 2 \Delta n}{k_{0}^{3} n_{d}^{3} L_{P D} \operatorname{Cos}\left(\Theta_{R}\right)} h
$$

Overall sensitivity depends on the interrogation method (intensity, wavelength or angular). The sharpness of the resonance peak also is a factor in determination of the sensitivity, most evident in the intensity measurement schemes. When a thin metal layer ( 30 to $100 \mathrm{~nm}$ ) is used in the grating coupler, whenever the plasmon is excited, it is partially re-radiated (transmitted) into the substrate (Fig. 1c). This plasmon enhanced transmission takes place for higher order resonant coupling or for non-zero azimuth excitation as well. Therefore, the transmission type device features the full benefits of grating coupled plasmon resonance.

Sensitivity optimization of transmission type devices are the same as reflection type grating coupled plasmon resonant devices. However, metal thickness has to be optimized as well. Near a resonance condition, the transmitted intensity can be approximated by

$$
T\left(\Theta_{i}, \Theta_{R}\right) \cong \frac{T_{0}}{1+4\left(\Theta_{i}-\Theta_{R}\right)^{2} / \Delta \Theta^{2}}
$$


where $\Theta_{\mathrm{i}}$ is the angle of incidence, $\Theta_{\mathrm{R}}$ is the resonance angle and $\Delta \Theta$ is the full-width at half-maximum (FWHM) of the resonance (Fig. 1d). In the intensity interrogation scheme, the illumination is incident at a fixed angle $\Theta_{\mathrm{i}}$ and transmitted intensity is monitored. Maximum SPR sensitivity is achieved when $\left|\partial T / \partial \Theta_{R}\right|$ $\sim 2 \mathrm{~T}_{0} / \Delta \Theta$, which takes place when $\Theta_{\mathrm{i}} \sim \Theta_{\mathrm{R}} \pm \Delta \Theta / 4$. Theoretical refractive index sensitivities of the first and second order resonances are $50^{\circ} / \mathrm{RIU}$ and $81^{\circ} / \mathrm{RIU}$ for $740 \mathrm{~nm}$ period grating in $n_{\mathrm{d}}=1.33$ fluid with an excitation wavelength of $632.8 \mathrm{~nm}$. Optimal grating depth is $30 \mathrm{~nm}$ and calculations assume $45 \mathrm{~nm}$ silver film. The FWHM of both the first and second order resonances are $\Delta \Theta \sim 2^{\circ}$ with a peak transmission of $T_{0}=0.3114$ for the first order and $T_{0}=0.167$ for the second order. Under these optimal conditions, assuming a $1 \mathrm{~mW}$ illumination and $0.4 \mathrm{~A} \mathrm{~W}^{-1}$ responsivity of the silicon readout photodiode, the first order resonance yields a current sensitivity of $6.2 \mathrm{~mA} / \mathrm{RIU}$. In this configuration, the photodiode current is calculated to be $62.3 \mu \mathrm{A}$ and the corresponding shot noise current is $4.46 \mathrm{pA} / \sqrt{\mathrm{Hz}}$. Therefore, shot noise limited minimum detectable refractive index is calculated to be $7.19 \times 10^{-10} \mathrm{RIU} / \sqrt{\mathrm{Hz}}$. This sensitivity level would require the measurement of current to 1 part in $1.4 \times 10^{7}$. If the precision of measurement is limited by electronics to 1 part in 65536 (16 Bits), the minimum detectable refractive index is calculated to be $1.53 \times 10^{-7} \mathrm{RIU} / \sqrt{\mathrm{Hz}}$. If the second order resonance is used, the reduction in the peak transmission compensates for the greater angular sensitivity ( 81 Deg/RIU for the second order compared to $50 \mathrm{Deg} / \mathrm{RIU}$ for the first order), and a similar refractive index resolution is obtained.

\section{Experimental}

The sensor assembly, photographed in Fig. 1a and schematically illustrated in Fig. 1b, has a sandwich structure of three integrated layers. On top, there exists a polymethyl methacrylate (PMMA) cover forming the flow channel. A photodiode substructure lying at the bottom serves as a detector and in between there is the patterned and metallised transparent polymer, i.e. the grating structure, to function as the surface plasmon (SP) coupler.

\section{Photodiode substrate fabrication}

In order to demonstrate that the device can be realized on standard silicon substrates with low-cost and widely available manufacturing equipment, the photodiode was fabricated using a metal induced crystallization procedure as described previously. ${ }^{22}$ First an amorphous silicon layer is deposited (in barebrown colour) on to an n-type silicon wafer (in purple) by using plasma enhanced chemical vapour deposition (Fig. 2a). An aluminium layer (with a mass thickness of $50 \mathrm{~nm}$ ) was deposited on-top using thermal evaporation and it was then annealed (in a $\mathrm{N}_{2}$ atmosphere at $500{ }^{\circ} \mathrm{C}$ for $30 \mathrm{~min}$ ) for partial crystallization and p-type doping of the n-type Si-wafer substrate (Fig. 2a). The aluminium residue on the surface, i.e. the unreacted aluminium after the partial induced crystallisation (due to thermal annealing), was cleaned by using hydrofluoric acid (HF) rinse (Fig. 2b). Then, an additional aluminium layer was deposited by thermal evaporation and the deposited layer was lithographically patterned for making contact pads for the p-type (aluminium, top) region (Fig. 2c). The contact pad for the n-type region was (a)

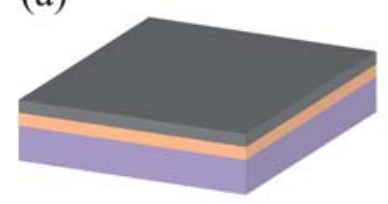

(c)

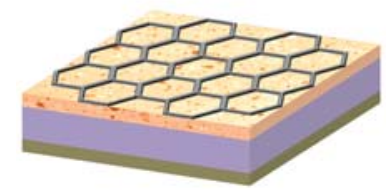

(e)

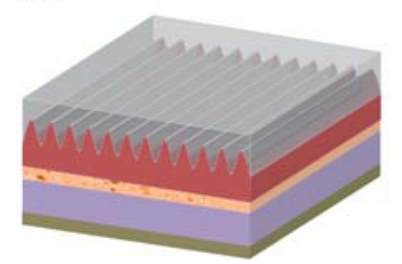

(b)

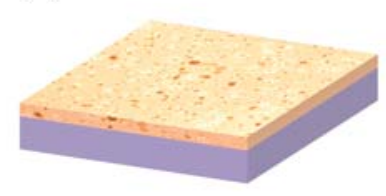

(d)

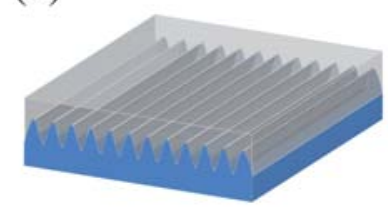

(f)

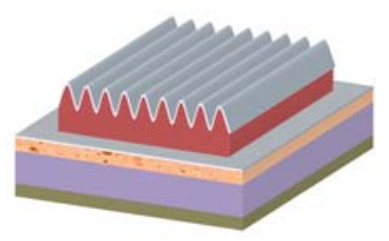

Fig. 2 Fabrication steps of a grating coupler-integrated photodiode ((a)-(f)). (a): n-type Si wafer, amorphous $\mathrm{Si}$, aluminium (from bottom to top). (b): Amorphous $\mathrm{Si}$ is aluminium doped during thermal curing. (c): Contact pads on top-side are patterned by lithography of an additionally deposited Al layer. Photodiode substrate is completed by the deposition of gold-palladium at the bottom-side to form the contact pads. (d): Grating profile is replicated from a surface modified DVD disc. PDMS is poured on the master mold and cured at $70{ }^{\circ} \mathrm{C}$ for $5 \mathrm{~h}$. Cured PDMS is peeled off from the master mold and used as elastomeric stamp. (e): Photoresist polymer is spin-coated on the photodiode substrate. Elastomeric stamp is released gently on the polymer film and hard-baked. Stamp is mechanically removed from the sample. (f): Contact mask is defined by the optical lithography. Thin Ag film is thermally evaporated to be used for plasmonic coupling.

made by deposition of a gold-palladium layer on the backside of the wafer (Fig. 2c). The device has also been fabricated using commercial photodiodes and it was observed to produce a similar performance.

\section{Nano-imprinted mould fabrication}

Gratings were replicated using the elastomeric molding technique by nano-imprinting. ${ }^{23,24}$ Previously, we have shown that modified optical disc surface profiles can be used to excite sharp and well defined plasmon resonances. ${ }^{25}$ Various tuned grating profiles were studied experimentally and theoretically (using rigorous coupled wave analysis, $\mathrm{RCWA}{ }^{26}$ ). For $\mathrm{n}_{\mathrm{d}} \sim 1.33$, the optimum grating structure has a groove depth of $30 \mathrm{~nm}$ with a tuned Ag film thickness of $45 \mathrm{~nm}$. The optimal topography and metal thickness produce the desired sharp resonance curves with high transmission. Thicker films reduce the transmission of stray light at the expense of reduced transmission of the signal. Gratings derived from Digital Video Disc (DVD) surfaces had a similar topographic corrugation $(45 \mathrm{~nm})$ and were used as the master mold. Polydimethylsiloxane (PDMS) (Slygard 184, Dow 
Corning) was poured on the substrates and cured at $70^{\circ} \mathrm{C}$ for $5 \mathrm{~h}$ (Fig. 2d). After curing, PDMS was peeled off from the master mold and used as an elastomeric stamp. A photoresist (AZ5214, AZ Electronic Materials) diluted $1: 3$ by a photoresist thinner (AZ1500, AZ Electronic Materials) was spin-coated at $8000 \mathrm{rpm}$ in order to form the grating material. Elastomeric stamp was then released softly on the thinned photoresist film and hardbaked on a hotplate at $120^{\circ} \mathrm{C}$ for about $5 \mathrm{~min}$. Finally, the stamp was mechanically removed from the sample (Fig. 2e). Metallization is done by thermally evaporation of silver onto the imprinted grating (Fig. 2f). The described procedure does not require external pressure to be applied to the PDMS stamp and results in uniform and distortion free gratings over large areas (greater than $10 \mathrm{~cm}^{2}$ ).

\section{Measurements}

Measurements were performed on an optical table with various $\mathrm{He}-\mathrm{Ne}$ laser sources $(2 \mathrm{~mW}$ and $8 \mathrm{~mW}$ nominal power) and a $650 \mathrm{~nm}$ laser diode ( $5 \mathrm{~mW}$ nominal power) was used to excite surface plasmon polaritons. Both TE and TM polarizations were measured, plasmon coupling is seen to occur for TM mode only. The grating direction is kept perpendicular to the plane of incidence and zero azimuthal angle was chosen. The angle of incidence (AOI) was stepped in the angular range of -50 and +50 degrees. In order to test the refractive index sensitivity of the device, distilled water (nominal refractive index 1.33) and a 5\% $\mathrm{NaCl}$ solution (nominal refractive index 1.342) were used. Liquid samples were streamed into the fluidic channel utilizing gravity. Photodiode outputs were monitored using precision current meters (Keithley 2400).

\section{Results and discussion}

\section{Grating profiles}

Grating surfaces were characterized by using atomic force microscopy (AFM) (Fig. 3a). In comparison to a uniform profile, a bi-harmonic surface profile enhances the second order coupling to the plasmon mode. Both resonances were observed in the reflection (in Fig. 3b) and transmission mode measurements (Fig. 3c). Plasmonic properties of silver coated gratings were characterized by measuring the reflection spectra using variable angle spectroscopic ellipsometry (using a J. A. Woollam, VASE system).

\section{Plasmon resonance characteristics and sensitivity}

We calculate the resonance angles for $632.8 \mathrm{~nm}$ excitation on a $\Lambda=740 \mathrm{~nm}$ grating surface, using silver as the metal layer. The first and second order resonances are calculated to occur at $\Theta_{\mathrm{R}}=33.9^{\circ}$ and $17.4^{\circ}$ for $n_{\mathrm{d}}=1.33$ (after correction for refraction at the air/cover interface). Theoretical resonance angles are shifted to $34.8^{\circ}$ and $16.6^{\circ}$ for $n_{\mathrm{d}}=1.342$ (representing $\mathrm{NaCl}$ solution). Experimentally measured resonance angles closely match the theoretical values. In DI water, the first and second order resonances are measured to occur at $\Theta_{\mathrm{R}}=34.1^{\circ}$ and $17.9^{\circ}$, while the angles shift to $\Theta_{\mathrm{R}}=35.2^{\circ}$ and $16.9^{\circ}$ for $\mathrm{NaCl}$ solution. Both resonances have FWHM of $\Delta \Theta \sim 1.5^{\circ}$. Contrary to theoretical calculations, the angular sensitivities of the resonance are
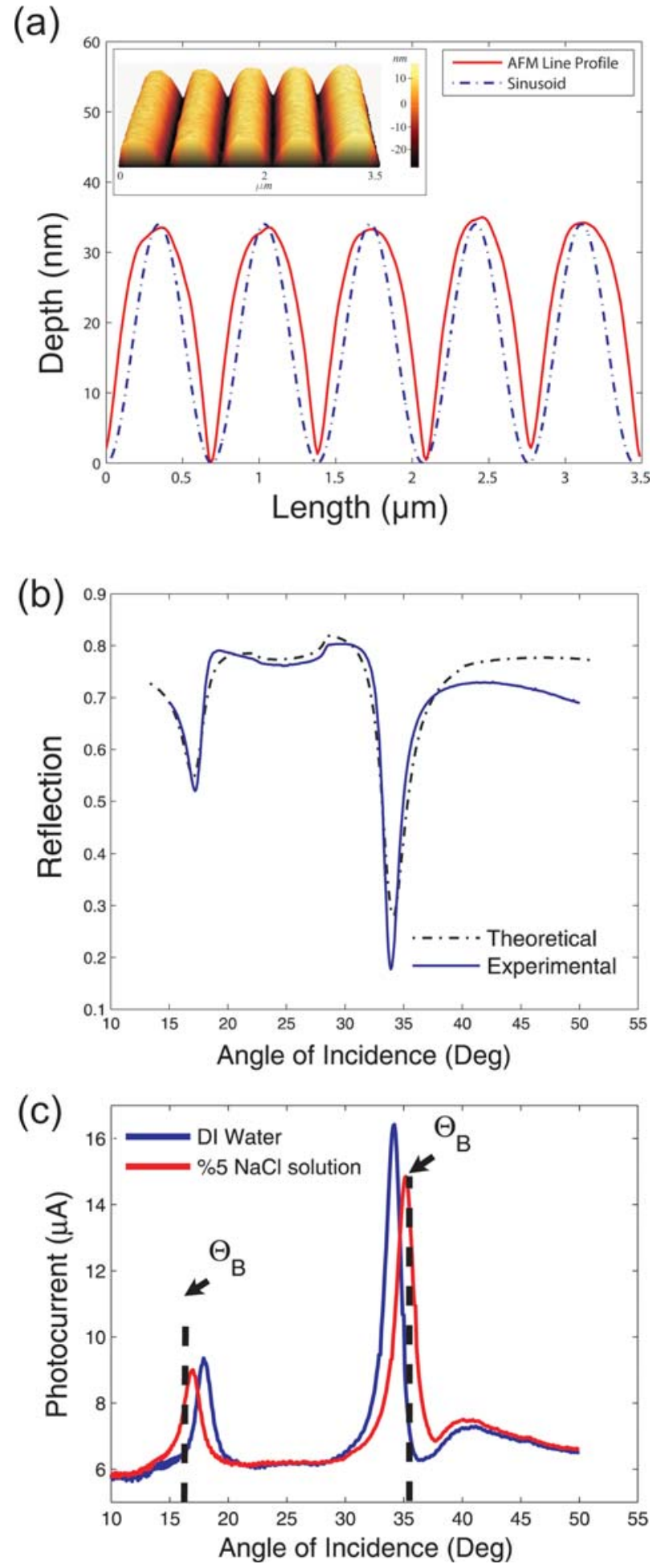

Fig. 3 (a) AFM line profile of the grating structure with a depth of $35 \mathrm{~nm}$ is depicted compared to a sinusoidal grating profile. Inset figure illustrates AFM topography of the corresponding grating structure with a groove depth of $35 \mathrm{~nm}$ and $45 \mathrm{~nm}$ thick silver coating. (b) Reflection behaviour of the grating structure is depicted in water medium under incidence of a $632.8 \mathrm{~nm}$ wavelength light source. Solid lines show the reflection measurement results whereas the dashed lines show theoretical results. (c) Measured photocurrent response of the integrated sensor as a function of the angle of incidence for water and $\mathrm{NaCl}$ solution. Bias angles used in the time-resolved measurements are shown by arrows. 
observed to be $91 \mathrm{Deg} / \mathrm{RIU}$ and $83 \mathrm{Deg} / \mathrm{RIU}$. The discrepancy may be due to systemic measurement errors of the resonance angles in our measurement setup. In time resolved measurements, DI water and $\mathrm{NaCl}$ solution were streamed through the fluidic channel and photocurrents were monitored (Fig. $4 \mathrm{a}$ and $4 b)$. The bias angles are chosen such that an increase of the refractive index causes an increase of the photocurrent $\left(\Theta_{i}=35.6^{\circ}\right.$ and $\Theta_{i}=17.1^{\circ}$, See Fig. 3c). The current sensitivity at the highest slope of the transmission curve is experimentally determined to be $\partial i / \partial \Theta_{R}=7.37 \mu \mathrm{A} / \mathrm{Deg}$ for the first order and $\partial i / \partial \Theta_{R}=2.92 \mu \mathrm{A} / \mathrm{Deg}$ for the second order. The resulting overall sensitivities can be then calculated as $\partial i / \partial n=6.11 \times 10^{-4} \mathrm{~A} / \mathrm{RIU}$

(a)

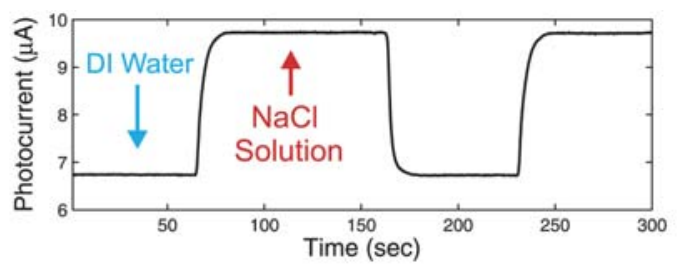

(b)

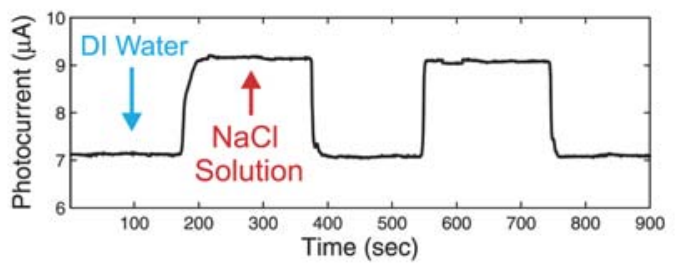

(c)
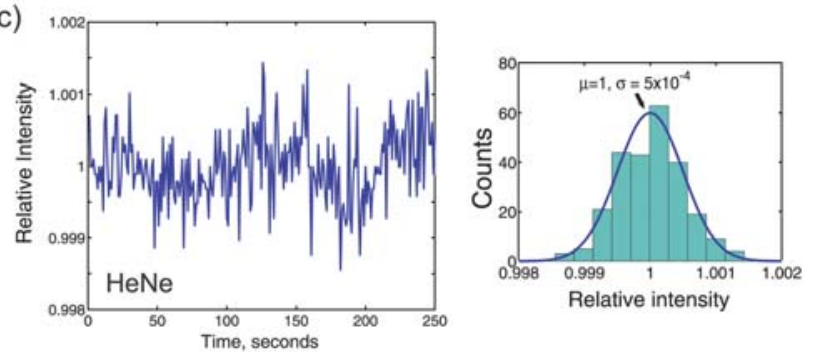

(d)

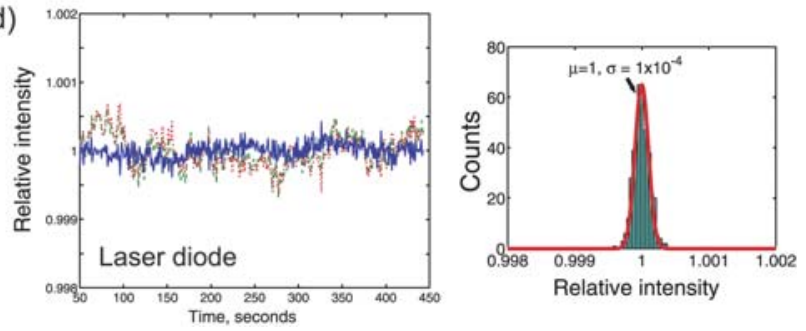

Fig. 4 Sensor responses to fluid exposures: (a) around the 2nd order resonance peak biased at $17.1^{\circ}$ and (b) around 1st order resonance peak biased at $35.6^{\circ}$ respectively. A $632.8 \mathrm{~nm} \mathrm{HeNe}$ laser source is used, resulting in an equivalent refractive index noise of $6.6 \times 10^{-6} \mathrm{RIU} / \sqrt{\mathrm{Hz}}$. (c) Stability of the measurement set-up using a $2 \mathrm{~mW}$ HeNe source and (d) using a power stabilized $650 \mathrm{~nm}$ laser diode. Further noise reduction is demonstrated using balanced detection. In (d) a reference photodiode monitors the light intensity at the source while the device is biased near the first order resonance. Normalized intensities for the reference diode (dotted) and SPR diode (dashed) are shown as well as the ratio of the two (solid). In the balanced measurement, the SPR intensity signal can be measured with a $\% 0.01 / \sqrt{\mathrm{Hz}}$ relative intensity noise. Resulting detection noise floor is $1.2 \times 10^{-6} \mathrm{RIU} / \sqrt{\mathrm{Hz}}$. Histograms of current readings are shown with corresponding Gaussian curve fits. for the first order and $\partial i / \partial n=2.42 \times 10^{-4} \mathrm{~A} / \mathrm{RIU}$ for the second order. The main reason for the observed reduced value of $\partial i / \partial n$ (compared to $\partial i / \partial n=6.2 \times 10^{-3} \mathrm{~A} / \mathrm{RIU}$ for the optimal design with $1 \mathrm{~mW}$ laser power) is that the thickness of the silver layer is about $130 \mathrm{~nm}$ (greater than the optimal value of $45 \mathrm{~nm}$ ). For the present device and assuming a shot-noise limited measurement, the minimum detectable refractive index change is estimated as $5.61 \times 10^{-9} \mathrm{RIU} / \sqrt{\mathrm{Hz}}$. However, due to laser source dominated noise during measurements (Fig. $4 \mathrm{c}$ ), this value was determined as $6.3 \times 10^{-6} \mathrm{RIU} / \sqrt{\mathrm{Hz}}$. The measured photocurrent noise level near DC is around $1.7 \mathrm{nA} / \sqrt{\mathrm{Hz}}$ as opposed to a theoretical shot noise level of $1.8 \mathrm{pA} / \sqrt{\mathrm{Hz}}$. Using a laser diode and an external reference photodiode, the transmitted intensity can be measured to an accuracy of 1 part in $10^{4}$, yielding a sensitivity of about $1.2 \times 10^{-6} \mathrm{RIU} / \sqrt{\mathrm{Hz}}$ (Fig. 4d).

\section{Discussion}

We demonstrated detection of changes in the RI of the streamed solutions (DI water and $5 \% \mathrm{NaCl}$ solution) with reversible, repeatable and stable sensor response, yielding an equivalent RI noise of $6.3 \times 10^{-6} \mathrm{RIU} / \sqrt{\mathrm{Hz}}$ (limited by the laser intensity noise and instrumental drifts). For the present device, optimization of the coupling condition may further enhance the sensitivity. It is well known that as the coupling angle $\Theta_{R}$ approaches grazing angles, the angular sensitivity increases. ${ }^{27}$ This fact is also predicted in eqn (5), as the angular sensitivity is dependent on $\Theta_{R}$, i.e. $\delta \Theta_{\mathrm{R}} / \delta_{\mathrm{Nd}} \propto 1 / \operatorname{Cos}\left(\Theta_{\mathrm{R}}\right)$. The sensitivity can be further enhanced by about an order of magnitude, by choosing a different grating period or excitation wavelength, so that $\operatorname{Cos}\left(\Theta_{\mathrm{R}}\right) \sim 0.1$. Also, recently Romanato et al. ${ }^{28,29}$ reported that azimuthal rotation of the grating results in a larger resonance angle shift (up to $800 \mathrm{Deg} / \mathrm{RIU}$ compared to $50 \sim 100 \mathrm{Deg} / \mathrm{RIU}$ for our present configuration). For optimal azimuthal excitations, a sensitivity improvement of more than an order of magnitude should be achievable in our device, compared to the same grating excited with zero azimuth angle. In such a case, the sensitivity is expected to be $\sim 10^{-7} R I U / \sqrt{\mathrm{Hz}}$ for a relative readout accuracy of $10^{-4}$. The shot noise limited detection limit also benefits from such an improvement and can be on the order of $1 \times 10^{-10} \mathrm{RIU} / \sqrt{\mathrm{Hz}}$ using $1 \mathrm{~mW}$ illumination. Although systemic errors lead to slight discrepancies between theory and experiment, the sensitivity is estimated to be on the correct order.

The device we present here is particularly appropriate for array detection and balanced detection. For example, by integrating the grating coupler onto an array detector (such as a CCD), multiple sensing spots can be monitored simultaneously. Using differential functionalization, balanced detection can be done within the same chip. Such an in situ balanced detection potentially eliminates temperature related drifts and renders the device insensitive to refractive index fluctuations of the working fluid.

Another way of performing array sensing is by using focused illumination. Such area-selective illumination allows a single large-area photodiode to be used to detect the resonance signal from a multitude of individually functionalized sensing spots. The well known relation between the focal spot size (D) and beam divergence $\left(\theta_{\mathrm{d}}\right)$ in diffraction limited focusing $(D=2 \lambda /$ $\left.\pi \mathrm{n}_{\mathrm{d}} \sin \left(\theta_{\mathrm{d}}\right)\right)$ determines the minimum allowed beam divergence for a given spot size. If a focused beam is used, an effective 
broadening of the resonance peak is observed, which reduces the sensitivity. For a divergence angle of about 1 degree, a spot diameter of about $20 \mu \mathrm{m}$ is achievable with $632.8 \mathrm{~nm}$ light in water. Such an angular dispersion is seen to degrade the sensitivity only by about a factor of two.

\section{Conclusion}

Using a straightforward, easy-to-implement fabrication procedure, we demonstrated the possibility of achieving very promising sensitivity and sensor responses through integration of the grating coupled plasmon detection mechanism with the optical sensor. Our approach yielded a low cost, SPR sensor which could offer a high level of miniaturization. The device features the full benefits of grating coupled plasmon resonance, with room for sensitivity enhancements to levels comparable with prism coupled sensing. The detection scheme can find applications in integrated lab-on-chip designs where label free detection is desirable.

\section{Acknowledgements}

This work was partially funded by TUBITAK 107T547, and supported by the State Planning Agency of the Turkish Republic Project UNAM.

\section{Notes and References}

1 X. D. Hoa, A. G. Kirk and M. Tabrizian, Biosens. Bioelectron., 2007, 23, 151-160.

2 Otto, Z. Phys., 1968, 216, 398.

3 Kretschmann, Z. Phys., 1971, 241, 313.

4 J. Homola, Chem. Rev., 2008, 108(2), 462-493.

5 J. Van Gent, P. V. Lambeck, H. J. M. Kreuwel, G. J. Gerritsma, E. J. R. Sudholter, D. N. Reinhoudt and T. J. A. Popma, Appl. Opt., 1990, 29, 2843.

6 M. L. Dakss, L. Kuhn, P. F. Heidrich and B. A. Scott, Appl. Phys. Lett., 1970, 16, 523.
7 M. Piliarik, M. Vala, I. Tichy and J. Homola, Biosens. Bioelectron., 2009, 24, 3430-3435.

8 E. Ouellet, C. Lausted, T. Lin, W. T. Yang and E. T. Lagally, Lab Chip, 2010, 10, 581-588.

9 K. Gurel, B. Kaplan, H. Guner, M. Bayindir and A. Dana, Appl. Phys. Lett., 2009, 94, 233102.

10 L. Salomon, F. Grillot, A. V. Zayats and Fornel F. De, Phys. Rev. Lett., 2001, 86(6), 1110.

11 H. J. Lezec, T. Thio and L. Pasteur, Opt. Express, 2004, 12(16), 36313646.

12 U. Schröter and D. Heitmann, Phys. Rev. B: Condens. Matter Mater. Phys., 1998, 58(23), 15419.

13 L. Pang, G. M. Hwang, B. Slutsky and Y. Fainman, Appl. Phys. Lett., 2007, 91, 123112.

14 A. Lesuffleur, H. Im, N. C. Lindquist and S. Oh, Appl. Phys. Lett., $2007,90,243110$.

15 T. W. Ebbesen, H. J. Lezec, H. F. Ghaemi, T. Thio and P. A. Wolff, Nature, 1998, 391, 667.

16 A. C. R. Pipino, R. P. Duyne and G. C. Schatz, Phys. Rev. B: Condens. Matter, 1996, 53(7), 4162.

17 P. Adam, J. Dostálek, O. Telezhnikova and J. Homola, Proc. Of SPIE, 2007, 6585, 65851Y-1.

18 A. Kocabas, S. S. Senlik and A. Aydinli, Phys. Rev. B: Condens. Matter Mater. Phys., 2008, 77, 195130.

19 C. Neipp, A. Beléndez, S. Gallego, M. Ortuño, I. Pascual and J. T. Sheridan, Optics Express, 2003, 11, 16.

20 H. J. Simon, D. E. Mitchell and J. G. Watson, Phys. Rev. Lett., 1974, 33(26), 1531-1534.

21 J. Homola, Springer-Verlag, 2006, vol. 4, (part I), DOI: 10.1007/ b100321.

22 Okan Oner Ekiz, Koray Mizrak and Aykutlu Dâna, ACS Nano, 2010, 4(4), 1851-1860.

23 A. Kocabas, A. Dana and A. Aydinli, Appl. Phys. Lett., 2006, 89, 041123 .

24 A. Kocabas, F. Ay, A. Dana and A. Aydinli, J. Opt. A: Pure Appl. Opt., 2006, 8, 85-87.

25 B. Kaplan, H. Guner, O. Senlik, K. Gurel, M. Bayindir and A. Dana, Plasmonics, 2009, 4(3), 237-243.

26 International Intellectual Group, Inc., PCGrate. http:// www.pcgrate.com.

27 Homola Jiri, Koudela Ivo and Yee S. Sinclair, Sens. Actuators, B, 1999, 54, 16-24.

28 F. Romanato, K. H. Lee, H. K. Kang, G. Ruffato and C. C. Wong, Opt. Express, 2009, 17, 14.

29 K. H. Lee, F. Romanato, H. K. Kang and C. C. Wong, Sens. Actuators, B, 2010, 148, 181-185. 